\title{
Efficient Bandwidth Allocation with Dynamic Coding for Quality of Service in CDMA based Wireless Communication System
}

\author{
Syed Shakeel Hashmi \\ Assistant Professor, ECE \\ Faculty of Science \& \\ Technology, IFHE University \\ Hyderabad
}

\author{
K.Kishore Kumar \\ Assistant Professor \& HOD, \\ ECE, Faculty of Science \& \\ Technology, IFHE University \\ Hyderabad
}

\author{
Syed Abdul Sattar, PhD. \\ Professor \& HOD, ECE \\ Royal Institute of Technology \& \\ Science, Chevella, \\ Hyderabad
}

\begin{abstract}
This paper presents an approach for improving the Quality of Service (QoS) of CDMA systems based on the proposed switch coding scheme at the encoding process of transmission and a bandwidth scheduling scheme for achieving high throughput services in multi-rate CDMA system. The paper presents a bandwidth scheduling scheme based on the available resources depending upon the demanded service bandwidth. To avoid the Multi-access Interference (MAI) due to simultaneous requests a code switching scheme is proposed. The QoS metrics were evaluated for the developed system and compared with the conventional approach.
\end{abstract}

\section{Keywords}

CDMA System, Switch Coding, Multi-access Interference, QoS Parameters, Throughput.

\section{INTRODUCTION}

Wireless communication has evolved a long range from the day of evolutions. Primitively where analog communications were used for the data transfer over a wireless channel, the resource consumption, system accuracy and efficiency were very low. To improve the performance of the communication system the technology moved from analog mode of communication to digital communication. In this development multi-access technique were observed to be an improved version of the current digital system. In multi access technique, code division multi-access technique (CDMA) is observed to be the most promising approach for efficient resource utilization and service compatibility. From the day of CDMA usage in wireless communication it is under the phase of improvement for better service compatibility and resource utilization. Resource allocation remains an issue with the increase in service demand, the interference level is probable to increase, and because of this the available coding system may get affected from its estimated accuracy resulting in heavy degradation in quality of service. As the demand for higher service compatibility increases the traffic rate over the channel will be increasing resulting in heavy multi access interference created due to simultaneous users. In next generation CDMA system this will be a major problem. Techniques based on adaptive code [1] and Adaptive bandwidth allocation [2] approaches were proposed to reduce the interference effect. To overcome the access effect under fading channel an signature based coding technique is suggested in [3] or by using spreading codes. The authors in [4] and [5] examine the rate-assignment problem for both single and multi-sector CDMA systems, leaving the issue of decentralized non-uniform rate-assignment unaddressed. The authors in [6] proposed algorithms that jointly optimize both transmit power and spreading gain. All suggested techniques were observed to be an optimal power allocation, In this paper it is focused on the development of an approach for interference rejection based on the modified format of the coding scheme used in CDMA system. With this approach a simpler and less computational approach for MAI reduction in CDMA system is proposed. The paper is further present in 5 sections, where in a brief review to the CDMA communication system is presented in section 2 . In section 3 the proposed switch coding scheme for interference rejection is presented. In section 4 bandwidth scheduling algorithm for throughput improvement is described. In section 5 QOS measures and parameters selection is outlined. The obtained simulation results are outlined in section 6 and the conclusions are presented in section 7.

\section{COMMUNICATION SYSTEM AGE}

CDMA is the most suitable multiple access transmission technology for Mobile Communications and all the 3rd Generation Mobile Communication. The main reason for the success of this technology is the huge increase in capacity and coverage of CDMA systems when compared to other analog (FM) or digital (TDMA) transmission systems due to higher bandwidth and coherent uplink detection. Transmission of code combined with the useful information requires the availability of a much greater radio frequency bandwidth than that required transmitting the information. This is the reason why one refers to Spread Spectrum Multiple Access. A conventional CDMA based communication system is designed for the evaluation of the suggested coding system. The system architecture used and the conventions made for the implementation are as outlined below.

The CDMA based Spread spectrum communication architecture is considered for the evaluation as shown above. The communication model (conventional architecture) consisting of a multiple user spread code transmitting unit takes multiple user inputs and spreads using coding sequence allocated in a sequential manner. 


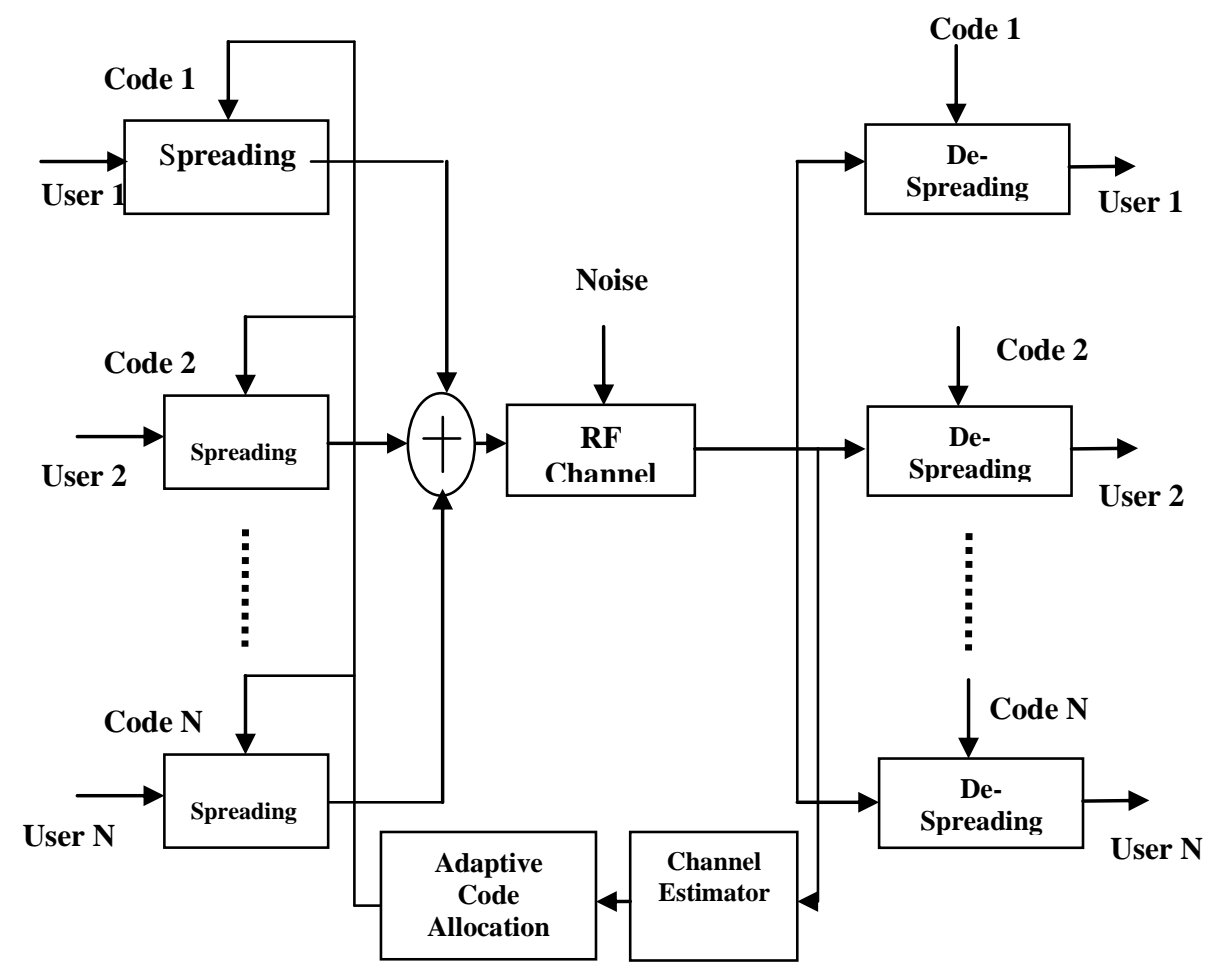

Fig 1: Conventional CDMA based communication system

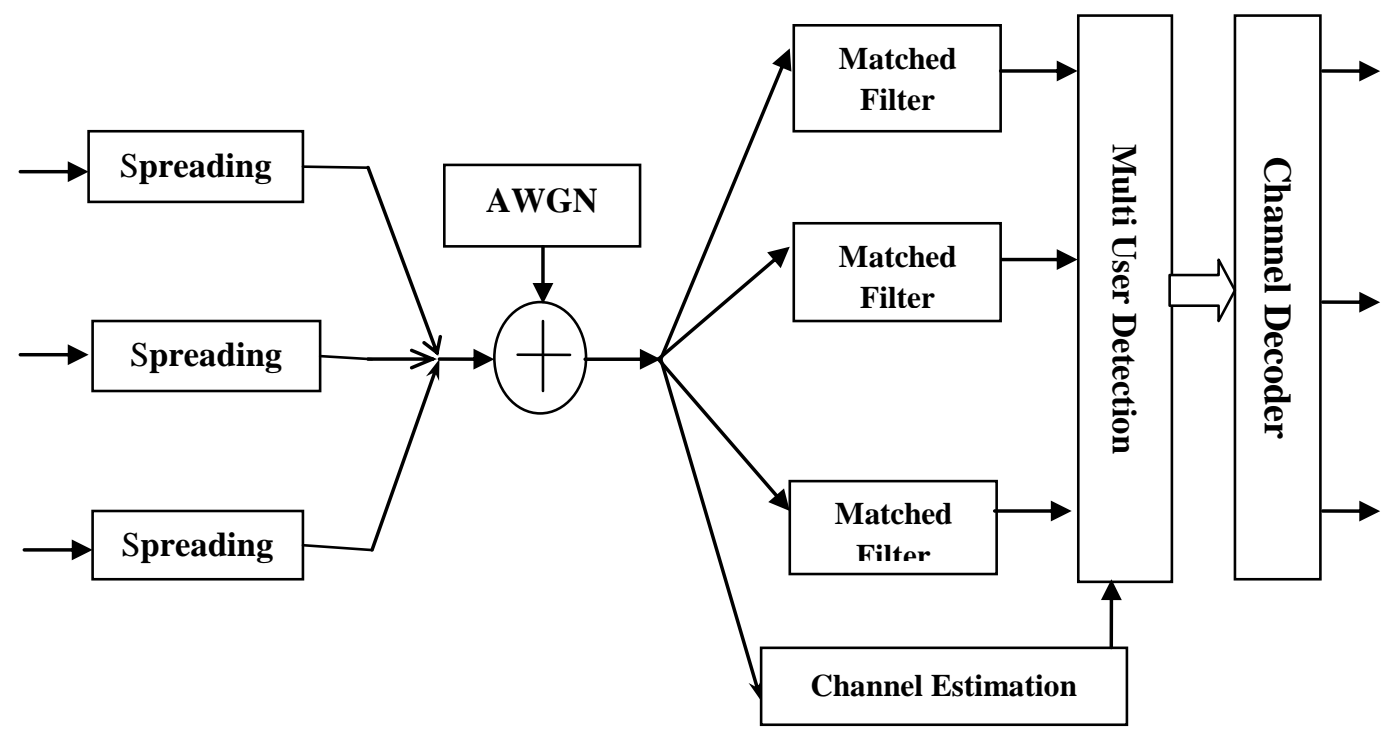

Fig 2: Suggested approach for switch code allocation

The practical problem that often arises in CDMA is the fact that the code sequences are not completely orthonormal, because they are chosen not orthonormal, to avoid capacity limitation, the signal coming from each user at the receiver has a random delay and the matched filter corresponding to one code will not totally suppress the interference caused by other signals. A conventional DS-CDMA system treats each user separately as a signal, while other users considered as noise or multiple access interference which is referred to as the near-far effect: Users near the base station are received at higher powers than those far away. Far away users suffer degradation in performance as the ratio of signal to interference is low. A tight power control is needed to alleviate this problem or one can use multi-user detection techniques. In multi user detection technique each user is detected in a sequential manner based on the reception of 
data. Due to channel interference the data in the channel are heavily corrupted there by estimation becomes difficult. Due to the sequential allocation of code at the transmission without the reference of channel effect is leading to heavy degradation in current CDMA based communication system. To overcome this issue an adaptive coding scheme is been suggested as explained in section 3 .

\section{CODE ALLOCATION SCHEME}

The use of any orthogonal code spreading sequences provides excellent performance for the downlink of DSCDMA systems in Additive White Gaussian noise (AWGN) channels. However, the hostile nature of the wireless channel can severely degrade the orthogonality of such sequences and unless compensated at the receiver it will result in significant multiple access interference (MAI). The MAI experienced by the different users depends on the cross correlations of the user's codes as well as the instantaneous values of the user's data symbols to be transmitted. The principle of the proposed technique is to exploit the dependency of multiple access interference on the instantaneous code allocation to the active users. For the allocation of code to active users a adaptive approach is developed as shown below.

A switch code allocation unit with channel estimator is incorporated with the conventional CDMA architecture. A switch code selection is carried out with preestimation processing in the transmission side for best code allocation.

The allocation algorithm is as outlined below, In the downlink transmission of a synchronous CDMA system of K users under frequency selective fading, where the channels' path delays are assumed to be an integer number of the chip period. All codes and channels are assumed to have normalized energy of one and length of $\mathrm{L}$ and $\mathrm{P}$ chips, respectively. The data frame is $\mathrm{N}$ symbols long. $\mathrm{Tb}$ and $\mathrm{Tc}$ are the symbol and chip periods, respectively. The received signal at the $\mathrm{u}^{\text {th }}$ Mobile Unit(MU) can be expressed as

$r_{u}(t)=\sum_{i=1}^{N} \sum_{k=1}^{K} \sum_{p=1}^{P} a_{k} x_{k}(i) c_{k}\left(t-i T_{b}-p T_{c}\right) h_{p u}(i)+n_{u}(t)$

where $x k(i)$, ak, ck are the $k^{\text {th }}$ user's PSK modulated data symbol for the $\mathrm{i}^{\text {th }}$ symbol period, amplitude and code, hpu(i) and nu(t) are the $\mathrm{u}^{\text {th }}$ MU's channel $\mathrm{p}^{\text {th }}$ tap coefficient and AWGN noise corrupting the signal of interest. The output of the Rake receiver of the $\mathrm{u}^{\text {th }}$ user can be expressed as

$$
\begin{aligned}
& d_{i u}=\sum_{p=1}^{P} \int_{i T_{b}+p T_{c}}^{(i+1) T_{b}+p T_{c}} r_{u(t)} h_{p u}^{*}(i) c_{u}^{*}\left(t-i T_{b}-p T_{c}\right) d t \\
& d_{i u}=a_{u} x_{i u}+I C I_{i u}+I S I_{i u}+M A I_{i u}+\eta_{i u}
\end{aligned}
$$

Here $x_{i u}$ is a compact representation of the desired $\left(u^{\text {th }}\right)$ user's signal for the $i^{\text {th }}$ period of interest, $\mathrm{MAI}_{\mathrm{iu}}$ is the cumulative MAI caused by the interfering $\mathrm{K}-1$ users and $\eta_{\text {iu }}$ is the noise component at the Rake output. If a discrete time representation is adopted by sampling the signals at the chip rate with rectangular pulses then $T_{b}, T_{c}$ can be omitted and the above terms can be defined as

$$
\begin{gathered}
M A I_{i u}=\sum_{\substack{n=-i \\
k \neq 1 \\
k \neq u}}^{N-i} a_{k} x_{i k} \sum_{l=1}^{L+P-1} s_{k u}[l] s_{u u}^{*}[l+n I] \\
=\sum_{\substack{n=1 \\
k \neq 1 \\
k \neq u}}^{N} a_{k} x_{i k} \rho_{k u n} \\
s_{k u}[l]=\sum_{p=1}^{P} c_{k}[l-p+1] \cdot h_{u}[p]
\end{gathered}
$$

Where $\mathrm{hu}[\mathrm{p}]$ is the discrete time representation of hpu(i) and

$$
\rho_{k u n}=\sum_{l=1}^{L+P-1} s_{k u}[l] s_{u u}^{*}[l+n L]
$$

is the cross correlation of the users multipart-corrupted signature waveforms $\left(\mathrm{S}_{\mathrm{ku}}\right)$. Evidently, even if orthogonal codes are used the resulting cross correlation of the codes viewed at the receiver is non zero due to the channel distortion. Majority of conventional precoding schemes, the knowledge of the channel response for all receivers is required at the base station (BS). This can be made available by channel estimation at the transmitter in the time division duplex (TDD) transmission mode, which is assumed in this paper. It can be seen channel state information (CSI) and data knowledge readily available at the BS the decision variables at the receiver can be pre-estimated. By selecting the appropriate code allocation for transmission at each symbol period the factors can be influenced and the distribution of the $\mathrm{d}_{\mathrm{iu}}$ values for all users can be improved to offer enhanced reliability in the detection. The detection can be made more reliable by optimizing the code-to-user allocation to be employed at each symbol period. In the proposed method the code allocation to be used is dynamically adjusted to the symbols $\mathrm{x}_{\mathrm{ik}}$ to be transmitted at the period $i$ of interest. Fine tuning of the users symbols and codes should be done so that the energy in the channel be used constructively instead of being wasted because of data misalignment as in conventional methods. As a result the effective received Signal to Interference and Noise ratio (SINR) can be increased and improved decision variables can be delivered at the Multi Users (MU) receivers without the need to increase the transmitted per-user-power. The selection of the optimum available code allocation pattern based on the instantaneous interference amongst users and the distribution of the resulting values of $d_{i}$. Since the performance of the worst user catalytically affects the overall system BER, the following code pattern selection criteria is proposed and examined here,

$$
\text { Sel_code }=\arg \max \left(\min \left(\hat{d}_{i, S}\right)\right)
$$

$\min \left(\hat{d}_{i, S}\right)$ determines the MU output that is the most prone to decision errors for each code allocation. From the $p_{c}$ available distributions of $\hat{d}_{i}$ according to the $\mathrm{P}_{\mathrm{C}}$ available 
code allocation patterns, the optimum is chosen as the one which maximizes the minimum of $\hat{d}_{i}$ that denotes the decision variable of the worst user at each symbol period for each distribution of $\hat{d}_{i}$. By maximizing the minimum of the decision variable distribution, the BER is minimized for the worst user. Hence the code allocation selected $\left(\mathrm{C}_{\mathrm{opt}}\right)$ is the one that delivers the highest decision variable for the worst user. In the case where two different allocation patterns offer the same minimum, the second minimum is considered. The stated switching approach is observed to minimize the interference effect but the other quality factor in CDMA system ie. throughput is also to be focused. To achieve the improvement in throughout to this system a resource scheduling scheme is also proposed. The method is as outlined below.

\section{BANDWIDTH SCHEDULING SCHEME}

In this algorithm a fairness based bandwidth adaptation algorithm is considered, which works in such a way that the allocated bandwidth to the ongoing calls will not differ from each other by more than one step. The bandwidth of an ongoing call is also allowed to be degraded below bandwidth requirement $b_{\text {req }}$ to minimize new call blocking and handoff call dropping probabilities. The operation is as outlined,

Let $\mathbf{w}_{\text {allc }}$ and $\mathbf{b}_{\text {allc }}$ denote the expected bandwidth for an incoming call and the bandwidth vector of ongoing calls, respectively. When a call arrives, the cell performs admission control by checking whether the total number of ongoing calls is less than the threshold t. If this condition is satisfied or if the incoming call is a handoff call, the cell tries to allocate maximum bandwidth to the incoming call; otherwise, the incoming new call is blocked. However, if the available bandwidth is not enough to allocate maximum bandwidth to an incoming call, the adaptation algorithm is invoked. The adaptation algorithm will randomly select an ongoing call with the current maximum bandwidth (i.e., max $\left.\left(\mathbf{b}_{\text {allc }}\right)\right)$ and de-grade allocated bandwidth of that call one step. At this point, expected bandwidth for incoming call increases one step. This operation is iteratively performed until the expected bandwidth for an incoming call is equal to the current minimum bandwidth of all ongoing calls (i.e., $\left.\min \left(\mathbf{b}_{\text {allc }}\right)\right)$. In contrast, if every call has the minimum bandwidth $b 1$, none of the ongoing call can be degraded. Therefore, an incoming call is dropped. For call thinning scheme, line 1 of this algorithm would be changed to admit the user

If ((incoming is a new call) and (number of ongoing calls $<\mathrm{K})$ \{

If (available bandwidth $>$ or $=b_{\max }$ ) Then assign $b_{\max }$ to incoming call

Else

$$
\begin{aligned}
& \left\{\quad \mathrm{b}_{\text {allocated }}=0\right. \\
& \text { for }(\mathrm{t}=1, \mathrm{t}<\mathrm{N}, \mathrm{t}++) \\
& \text { While }\left(\mathrm{b}_{\text {allocated }}<\mathrm{b}_{\min } \text { and } \mathrm{n}_{\mathrm{t}}>0\right) \\
& \{
\end{aligned}
$$

randomly degrade one of $\mathrm{n}_{\mathrm{t}}$ connections

by amount of $b_{\text {degrade }}$

\}

$$
\left.\begin{array}{l}
\mathrm{b}_{\text {degrade }}=\min \left(\mathrm{b}_{\min ,}, \mathrm{b}_{\mathrm{t}}-\mathrm{b}_{\min }\right) \\
\mathrm{b}_{\text {allocated }}=\mathrm{b}_{\text {allocated }}+\mathrm{b}_{\text {degrade }}
\end{array}\right\}
$$

\}

Else

Reject incoming call

\section{QoS MEASURES AND PARAMETER SETTINGS}

The aim of the work is improve the quality of service of the system. Hence the proposed system has come up with two algorithms Switch code algorithm and Resource scheduling scheme. Three QoS measures, namely Degradation ratio, Throughput, Degradation bandwidth is considered. These QoS measures can be calculated from total bandwidth which occupies in the channel. In wireless channel bandwidth is a scarce resource. Channel can accommodate only few users. If a new user wants to enter in the network due to lack of available bandwidth the request cannot be accepted. In this model by degrading the quality of service of existing users a new request can be accepted. These quality of service measures based on the above algorithm are measured.

Degradation ratio (DR): The fraction of time a user receives degraded QoS. Since reconsidering multi level QoS system DR is defined as

$$
D R=\frac{\sum_{i} \frac{\left(W_{\max }-W_{i}\right)}{W_{\max }} T_{i}}{\sum_{i} T_{i}}
$$

a user receives level-i QoS for Ti seconds.

Throughput: It gives the account of number of packets arrived at receiver. It measures the efficiency of system.

Throughput $=($ number of bits received $/$ number of bits send)*100

Degraded bandwidth (DB): It is measure of amount of bandwidth degraded from existing users. If number of users enters in the channel are increased then bandwidth degraded is increase. The degradation is stops for a user if he reaches the minimum bandwidth (b1)

For the simulation three simultaneous user data is considered. These data are spread with pseudo random noise sequence of spread factor equal to 31. Orthogonal Variable Spreading Factor (OVSF) spreading is used to spread the data with the spreading factor equal to 8 . This data is modulated using BPSK modulation. This is pass band transmission model. Two groups in the network are considered. We have presented an analytical framework for adaptive bandwidth allocation in cellular mobile networks. The numerical results obtained from the model have shown that the ABA can minimize handoff call dropping 
probability, while some calls might experience service degradation below an acceptable level. It is assumed that the 6 users are in the channel having bandwidth that is sufficient for all of them. But allocated bandwidth for all users is different. Assuming total bandwidth is $\mathrm{W}$.

Assuming first 2 users having allocated bandwidth (b1) $=30 \%$ of $\mathrm{W}$

Third user having allocated bandwidth (b2) $=25 \%$ of W

$4^{\mathrm{h}}$ and $5^{\text {th }}$ users having allocated bandwidth (b3) $=20 \%$ of W

$6^{\text {th }}$ user having allocated bandwidth (b4) $=15 \%$ of W

Available bandwidth $=7 \%$ of $\mathrm{W}$

Minimum bandwidth required for a call is equal to $15 \%$ of W. If a new user requests to enter in the channel then first two users bandwidth is degraded by $15 \%$ and this bandwidth is allocated to new user. But quality of first two users is degraded by $15 \%$ of W. In a system with degradable QoS, a user may receive different levels of QoS during the entire duration of his connection, depending on the loads of cells he traverses. Even if a user receives the highest level of QoS when he is admitted to a cell, the QoS may still be degraded when some other base stations on his "path" decide to degrade his QoS in order to accept more users. The prime consideration is interested in quality-degradable connections as long as the resultant quality is within the user specified QoS profile. The only QoS requirement discussed here is the bandwidth. For example, it can be a video streaming application with multiple transmission rates depending on the encoding schemes and resolution. It is assumed that there are 4 different quality levels. The bandwidth requirement of the ith quality level is denoted as bi and b1>b2>b3 $>b 4$. With such a degree of freedom, a base station may try to degrade the quality levels of some existing users in order to admit more users so as to improve the overall system performance. For example, it may be able to achieve high bandwidth utilization and maintain a small blocking and/or forced termination probability. if a user receives level-i QoS for Ti seconds

Assuming $\mathrm{T} 1=(\mathrm{W} /(\mathrm{b} 1 * 100)) * 10$;

$$
\begin{aligned}
& \mathrm{T} 2=(\mathrm{W} /(\mathrm{b} 2 * 100)) * 10 ; \\
& \mathrm{T} 3=(\mathrm{W} /(\mathrm{b} 3 * 100)) * 10 ; \\
& \mathrm{T} 4=(\mathrm{W} /(\mathrm{b} 4 * 100)) * 10 ;
\end{aligned}
$$

The above bandwidth allocation is achieved using adaptive bandwidth allocation method. If another user requests to enter in the network the same algorithm is applied to improve channel utilization but degradation of existing users increased. This algorithm can be applied until every user achieves minimum bandwidth (b1).

An analytical framework for adaptive bandwidth allocation in cellular mobile networks is presented. Using the framework, various performance metrics bandwidth, degradation ratio, propagation delay, throughput) have been observed.

\section{SIMULATION RESULTS}

For the evaluation of the proposed switch code allocation and fixed code allocation in CDMA system, Matlab modeling is developed and evaluated with varying SNR, offered load and channel fading factor. Matlab simulations for various combinations of the proposed technique with conventional methods have been performed for fading channels with additive white Gaussian noise. Random codes have been used to allow for the worst data detection case and to illustrate performance improvement when fixed code performance is poor.

The simulation evaluations are carried out at different performance metric of Low_SNR $=8$, Average_SNR $=15$, High_SNR $=22$, Low_load $=50$, Average_Load $=250$, High_Load $=500$, High_Fading $=1 / 2500$, Average_ Fading $=1 / 8000$, Low $\_$Fading $=1 / 10000$.

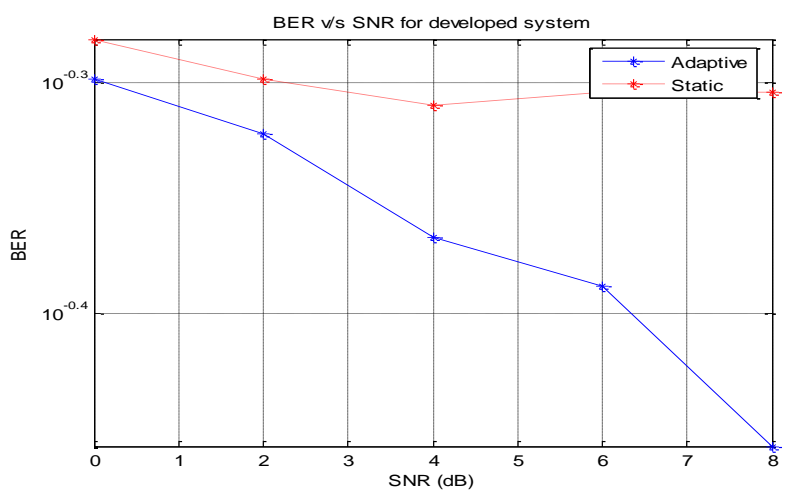

Fig 3: Overall system BER vs.SNR at $\mathrm{SNR}=8 \mathrm{~dB}$, $\mathrm{Load}=50$, Fading=1/10000

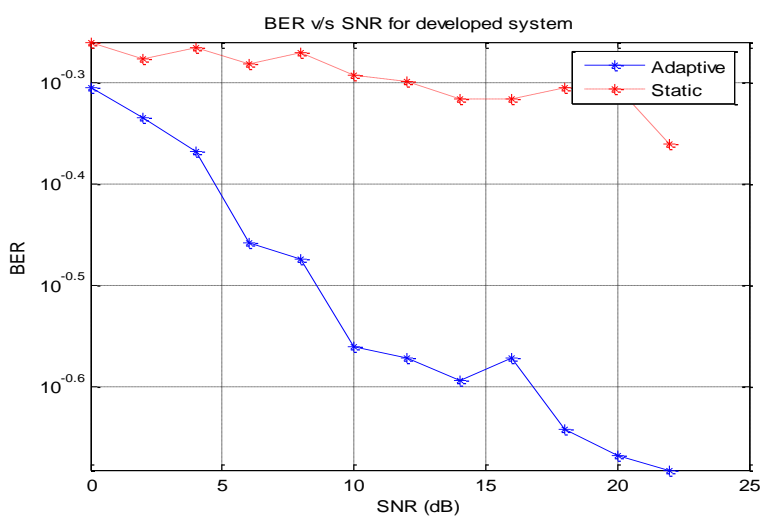

Fig 4: Overall system BER vs.SNR at $\mathrm{SNR}=8 \mathrm{~dB}$, Load $=100$, Fading $=1 / 10000$ 


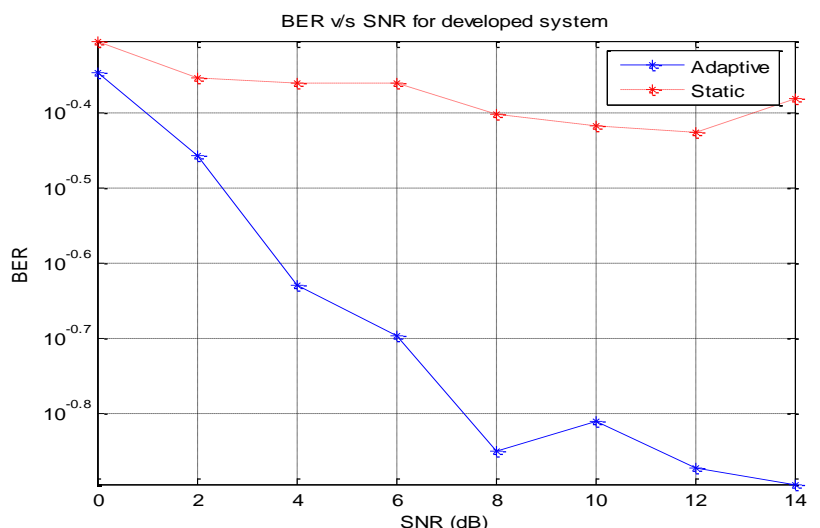

Fig 5: Overall system BER vs.SNR at $\mathrm{SNR}=8 \mathrm{~dB}$, Load $=250$, Fading $=1 / 10000$

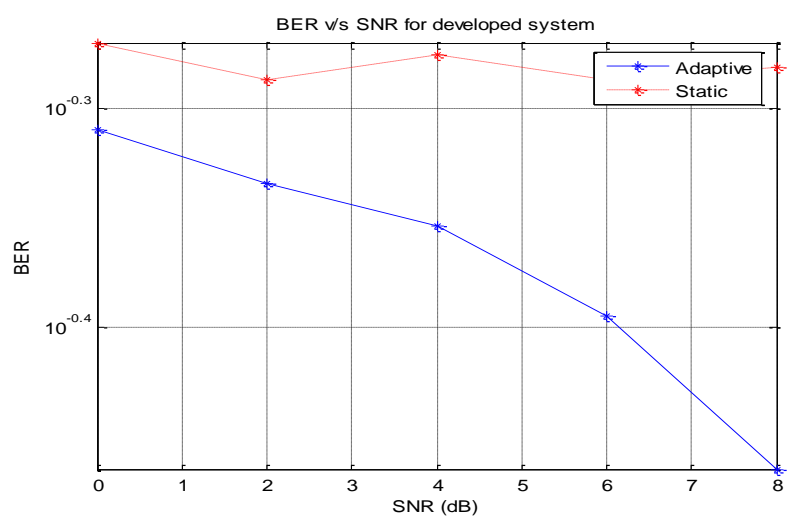

Fig 6: Overall system BER vs.SNR, SNR=28dB, $\mathrm{Load}=50$, Fading $=1 / 8000$

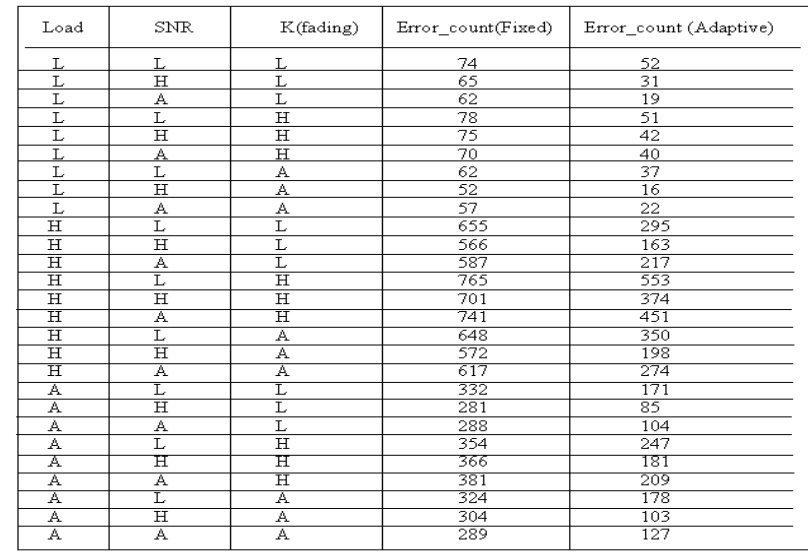

Table 1: Estimation error for the two methods developed under different load condition

For the proposed switch code allocation and fixed code allocation comparison is done with respect to the number of errors. In case of fixed code allocation the number of errors are always higher than switch code allocation.

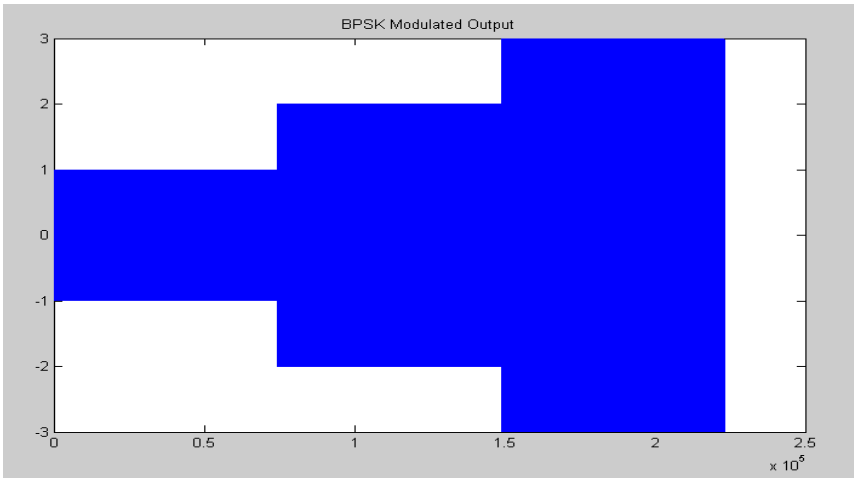

Figure 7 BPSK modulated output

Fig 7 shows the BPSK modulated data bundled with various power levels used before transmission. The three user data are modulated with sinusoidal carrier of o to $(2 *$ pi) sampled at 100 points. The user bandwidth data are processed for transmission over the wireless channel.

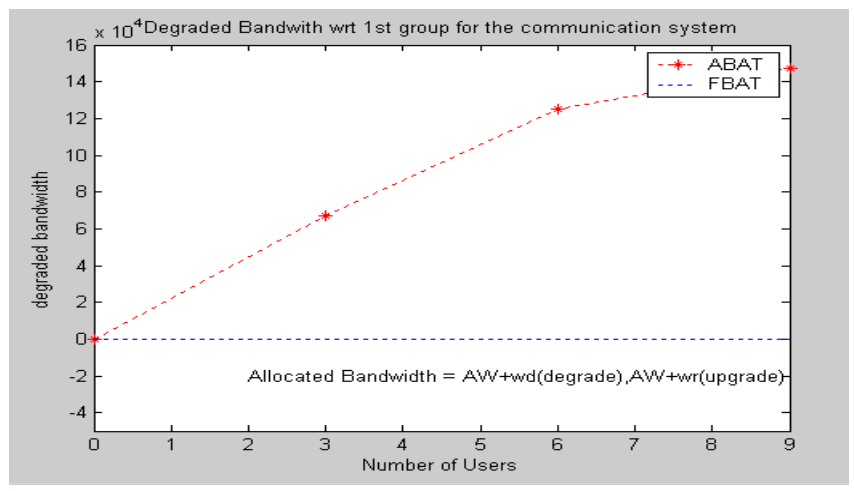

Fig 8: Bandwidth Degradation plot for the communication system

Figure 8 illustrates the performance plot for degradation in bandwidth allocated with respect to increase in number of users for fixed bandwidth allocation technique (fbat),adaptive bandwidth allocation technique (abat). The plot illustrates with increase in number of users the degradation eventually increases number for abat where as the fbat method the degradation is not applicable.

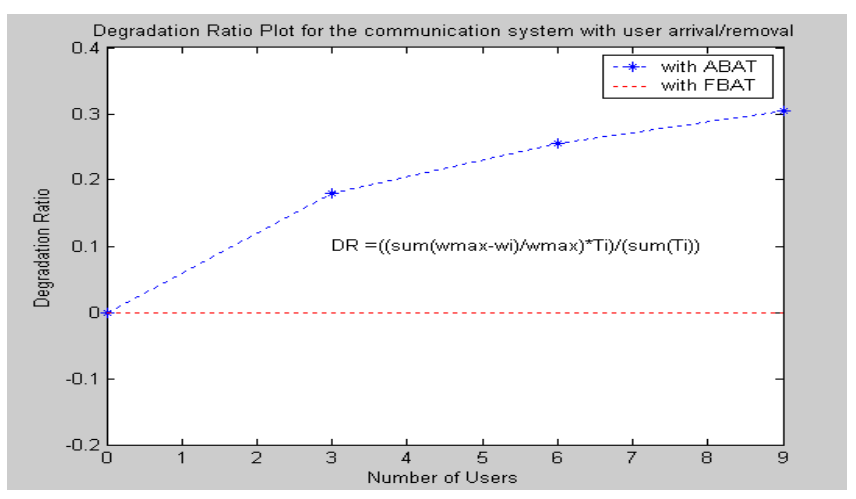

Fig 9: Degradation ratios for the implemented communication system 
Figure 9 shows the degradation the proposed two methods ABAT, FBAT with respect to group of users in the plot it is observed that the degradation for abat system varies whereas remain constant in case of FBAT system.

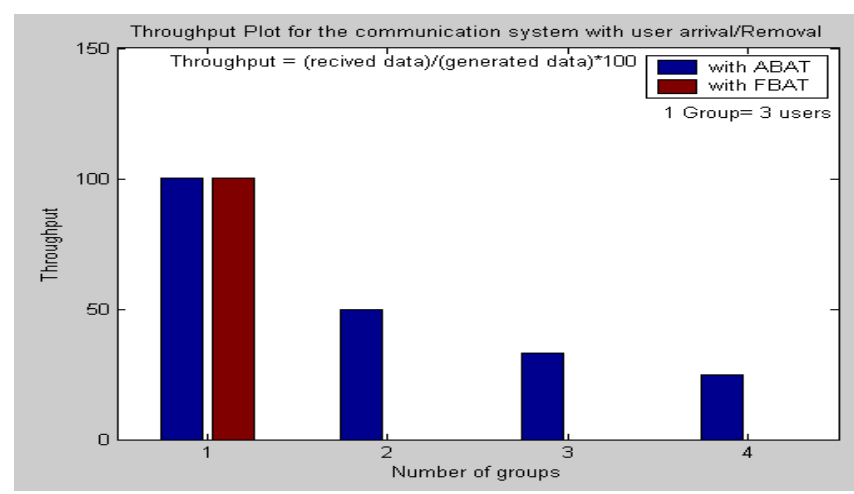

Fig 10: Throughput plot for the FBAT and ABAT system

Figure 10 shows the throughput analysis for two systems namely ABAT, FBAT methods. In case of ABAT method, it could be observed that the throughput remains decreased with increment in number of users where as it could be completely eliminated in case of FBAT system.

\section{CONCLUSION}

An adaptive approach for code allocation and bandwidth scheduling for throughput improvement are developed for a CDMA based communication system. The approach of code allocation is developed with a priorie estimation of channel in transmission and an adaptive allocation scheme is integrated for the estimation and synchronization in the transiver unit. The concept of switch code allocation in the communication system is observed to out perform the estimation accuracy in case of a fixed allocation scheme under variable channel condition. The paper suggested approach for CDMA communication used for interference rejection in highly bursty traffic conditions. A symbol to symbol switch code allocation is developed for the current CDMA architecture, It is observed that with the adaptive allocation of the coding sequence the system performance with respect to estimation accuracy is improved .The effect of estimation accuracy is also effective with respect to the offered load for the communication system. The Qos parameters are observed for throughput improvement and error reduction in the developed system.

\section{REFERENCES}

[1] Emad Alsusa " Adaptive Code allaocation for interference Management on the Downlink of DSCDMA Systems", IEEE transactions on Wireless communications, vol. 7, No. 7, July 2008.

[2] Chung-Tung Chou ,Kang G.Shin “ Analysis of Adaptive Bandwidth allocation in wireless Networks with Multilevel Degradable Quality of Service", IEEE transactions on Mobile computing, Vol. 3, No. 1, January-March 2004

[3] Gregory w. Wornell, "Spread-Signature CDMA: Efficient Multiuser Communication in the Presence of Fading", IEEE transactions on information theory, Vol. 41, No. 5, Sept 1995.
[4] S. Chakravarty, R. Pankaj, and E. Esteves, "An algorithm for reverse traffic channel rate contol for cdma2000 high rate packet data systems" , In Proceedings of IEEE Globecom, San Antonio, TX, 2001.

[5] E. Esteves, "On the reverse link performance of CDMA 2000 high rate packet data systems", In Proceedings of IEEE International Communication Conference, 2002

[6] S.J. Oh, D. Zhang, and K.M. Wasserman, "Optimal resource allocation in multiservice CDMA networks", IEEE Transactions on Wireless Communications, 2(4):811-821, July 2003

[7] Branimir R., and Won Mee Jang, "Transmitter Precoding in Synchronous Multiuser Communications", IEEE transactions on communications, Vol. 46, No. 10 , Oct 1998.

[8] Maite Brandt Pearce, and Anupama Dharap, "Transmitter-Based Multiuser Interference Rejection for the Down-Link of a Wireless CDMA System in a Multipath Environment", IEEE journal on selected areas of communication, Vol. 18, No. 3, March 2000.

[9] S.Shakeel Hashmi "Congestion Avoidance in IP based CDMA Radio Access Network", International Journal of Wireless and Mobile Networks,Vol.3, No.1, Febrauary 2011

[10] D. Zhang, S.J. Oh, and N.T. Sindhushayana, "Optimal resource allocation for data service in CDMA reverse link", In Wireless Communications and Networking Conference, volume 3, pages 1601-1606, 2004.

[11] C. Zhou, M.L. Honig, and S. Jordan, "Utility-based power control for a twocell CDMA data network", IEEE Transactions on Wireless Communications, 4(6):2764-2776, November 2005

[12] S. Sen, J. Jawanda, K. Basu, and S. Das, "Quality-of Service Degradation Strategies in Multimedia Wireless Network," Proc.IEEE Vehicular Technology Conf., vol. 3, pp. 1884-1888, May 1998.

[13] S. Singh, "Quality of Service Guarantees in Mobile Computing," Computer Comm., no. 19, pp. 359-371, 1996.

[14] M.R. Sherif, I.W. Habib, M.N. Nagshineh, and P.K. Kermani,"Adaptive Allocation of Resources and Call Admission Control for Wireless ATM Using Generic Algorithm," IEEE J. Selected Areas in Comm., vol. 18, no. 2 , pp. 268-282, Feb. 2000.

[15] T. Kwon, Y. Choi, C. Bisdikian and M. Naghshineh, "Call Admission Control for Adaptive Multimedia in Wireless/Mobile Network," Proc. First ACM Int'l Workshop Wireless Mobile Multimedia,pp. 111-116, Oct. 1998

[16] S. Choi and K.G. Shin, "Location/Mobility-Dependent Bandwidth Adaptation in QoS-Sensitive Cellular Networks," Proc. IEEE Vehicular Technology Conf., vol. 3, pp. 1593-1597, 2001.

[17] Y.B Lin, S. Mohan, and A. Noerpel, "Queuing Priority Channel assignment Strategy for PCS Handoff and Initial Access," IEEE Trans. Vehicular Technology, vol. 43, no. 3, pp. 704-712, Aug. 1994. 
[18] D.R.Srinivas,K.E.Sreenivasa Murthy, "Adaptive Code to user allocation for Interference Management on the Downlink DS-CDMA Systems", International Journal of Engineering Science and Technology, Vol.2(12),2010, 7641-7652

\section{AUTHOR'S PROFILE}

Syed Shakeel Hashmi received his B.E. Degree in Electronics \& Telecommunication Engineering from, B.A.M. University Aurangabad. India in 1999. He has obtained M.E. in Electronics \& Communication Engineering from, Osmania University, Hyderabad, India in 2006. He is a Research Scholar of Jawaharlal Nehru Institute of Advanced Studies, Secunderabad. He is currently working as assistant professor in the dept. of Electronics and Communication Engineering at FST, ICFAI Foundation for Higher Education (A Deemed University) Hyderabad India. His areas of interest are Wireless Communication, Heterogeneous wireless networks.

K.Kishore Kumar received his B.E. Degree in Electronics \& Communication Engineering from Koneru Lakshmaiah College of Engineering, Andhra Pradesh, India in 2000. He has obtained M.Tech in Digital Systems \& Computer Electronics from JNTU College of Engineering, Anantapur, India in 2004. He is currently working as a Head of
Department \& Assistant Professor in ECE Department at Faculty of Science \& Technology, ICFAI Foundation for Higher Education (A Deemed University), Hyderabad India. He has about 11 years of experience in teaching and industry together His areas of interest are Wireless Communication, Image Processing and VLSI.

Dr. Syed Abdul Sattar, is presently working as a Dean of Academics \& Professor of ECE department, RITS, Chevella, Hyderabad. He has completed his B.E. in ECE in 1990 from Marathwada University Aurangabad, M. Tech. in DSCE from JNTU Hyderabad, in 2002, and did his first Ph.D. from Golden state University USA, with Computer Science in 2004, and second Ph.D. from JNTU Hyderabad, A. P. India with ECE in 2007. His area of specialization is wireless communications and image Processing. He has about 21years of experience in teaching and industry together and recipient of national award as an Engineering Scientist of the year 2006 by NESA New Delhi, India. He has about 73 publications in International and National Journals and conferences.Presently he is guiding research scholars in ECE and Computer Science from different Universities. He is a member of Board of studies for a central university and reviewer/editorial member/chief editor for national and International journals. 